\title{
Procedimentos de Vineberg: proposta de uma variação técnica
}

\author{
José Glauco LOBO FILHO*
}

RBCCV 44205-533

\begin{abstract}
Lobo Filho J G - Procedimento de Vineberg: proposta de uma variação técnica. Rev Bras Cir Cardiovasc 2001; 16(1): 66-9.

RESUMO: O trabalho tem o objetivo de apresentar uma nova sistematização técnica para o procedimento de Vineberg, eficaz e facilmente reprodutível. Consta da introdução de um longo segmento da artéria torácica interna esquerda no miocárdio ventricular, utilizando-se uma "Bainha Introdutora".

DESCRITORES: Revascularização miocárdiaca, métodos. Procedimentos cirúgicos cardiopulmonares, métodos. Circulação extracorpórea. Artéria torácica esquerda, cirurgia. Artéria torácica interna, transplante.
\end{abstract}

\section{INTRODUÇÃO}

Várias publicações mostram que o procedimento de Vineberg é eficaz do ponto de vista funcional (1-4). Os principais motivos pelos quais foi suplantado não estão relacionados com a sua eficácia, mas sim, pelo grande impacto causado pela revascularização direta do miocárdio e a dificuldade imposta pela técnica originalmente descrita ${ }^{(5)}$. No início da década de sessenta, variantes técnicas propostas por SEWELL et al. ${ }^{(6)}$ e FAVLORO et al. ${ }^{(7)}$ procuraram dar maior aplicabilidade ao procedimento inicialmente proposto por Vineberg.

\section{MÉTODO}

Dentro dessa linha de pensamento, desenvolvemos uma variante técnica para realização do procedimento de Vineberg, que permite o implante da artéria torácica interna esquerda (ATIE) no miocárdio ventricular esquerdo de uma forma facilmente reprodutível e eficaz.
Após a dissecção da ATIE da maneira convencional com todo seu pedículo, aproximadamente sete centímetros de sua porção distal são inteiramente esqueletizados, com secção dos ramos intercostais em suas origens (Figura 1). Compressas são coloca-

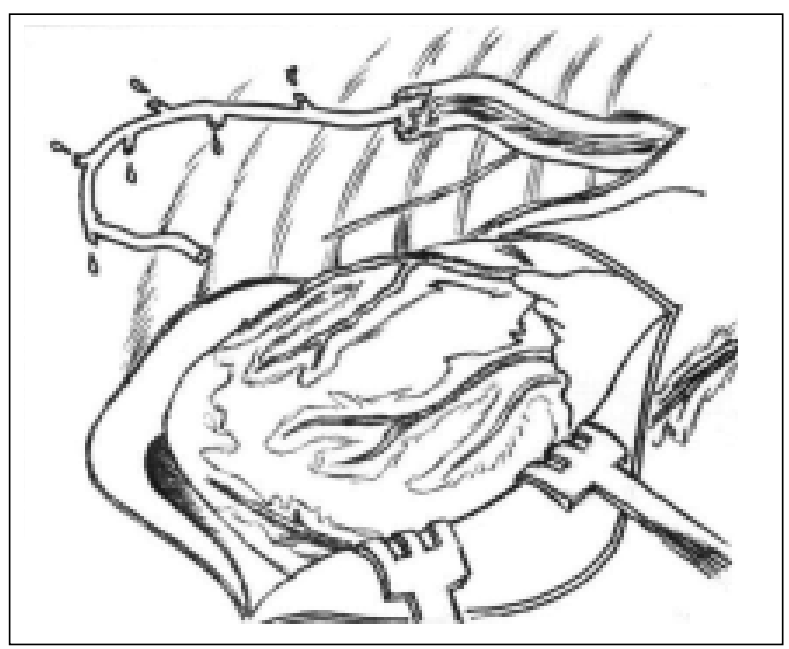

Fig. 1

Trabalho realizado no ICORP - Hospital Prontocárdio e Hospital Antônio Prudente. Fortaleza, CE, Brasil.

*Do Hospital Prontocárdio e do Hospital Antônio Prudente - Fortaleza.

Recebido para publicação em abril de 2001.

Endereço para correspondência: José Glauco Lobo Filho. Rua Dr. José Lourenço 625 - CEP: 60.115-280; Fortaleza, Ceará, Brasil. e-mail: glaucolobo@uol.com.br 
das na porção posterior do saco pericárdico de maneira que haja boa exposição da face anterior do ventrículo esquerdo (VE). Utilizando-se um "kit" introdutor para eletrodo endocárdico de marcapasso definitivo número "10F", transfixa-se com a agulha a porção do VE em que se deseja implantar a ATIE, geralmente paralela à artéria descendente anterior (DA) (Figura 2). O fio-guia é então posicionado no lugar da agulha, através do qual se passa a "bainha introdutora" (Figura 3). Em seguida, o fio-guia é posicionado dentro da bainha, cuja extremidade do referido fio-guia é ligada à porção distal da ATIE (Figura 4). O fio-guia é tracionado trazendo consigo a ATIE para dentro da bainha, que, depois de retirado, deixa a ATIE no túnel (Figura 5). Finaliza-se com a ligadura da porção distal da ATIE, fechando-se o orifício de saída na parede do VE e fixando-se o pedículo da referida artéria no epicárdio, vizinho ao orifício de entrada na parede do VE (Figura 6). Lembramos que, como originalmente descrita, esta cirurgia é realizada sem o uso do sistema de circulação extracorpórea.

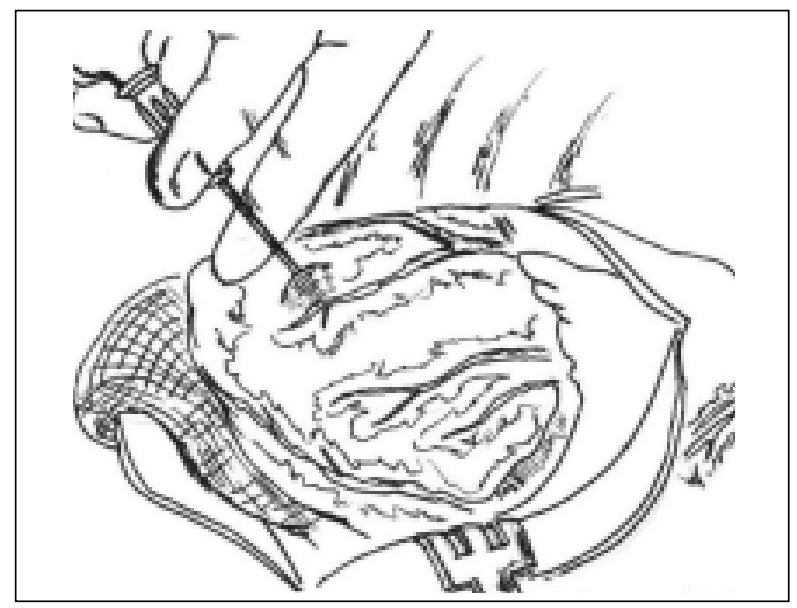

Fig. 2

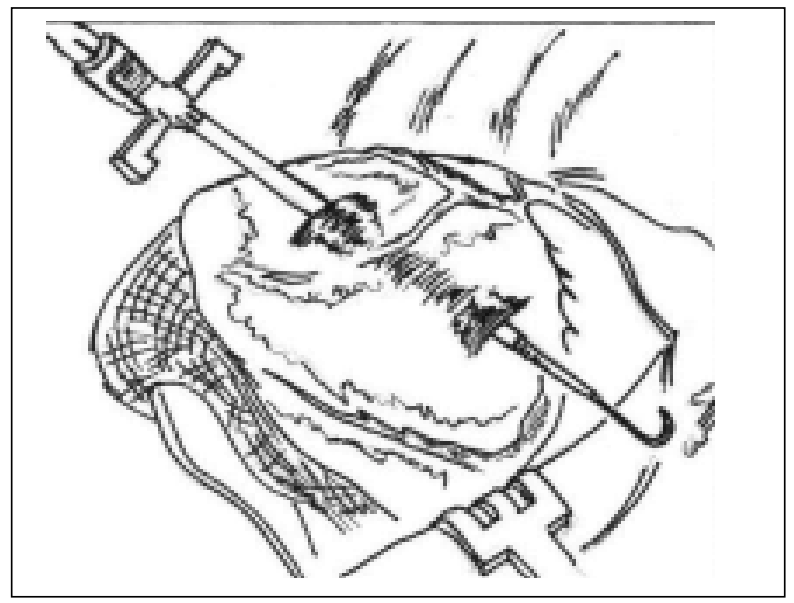

Fig. 3

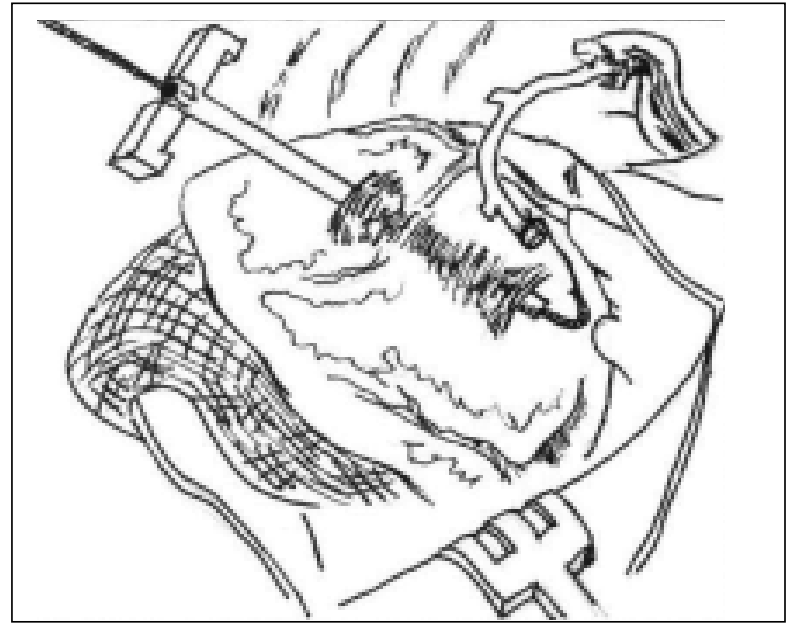

Fig. 4

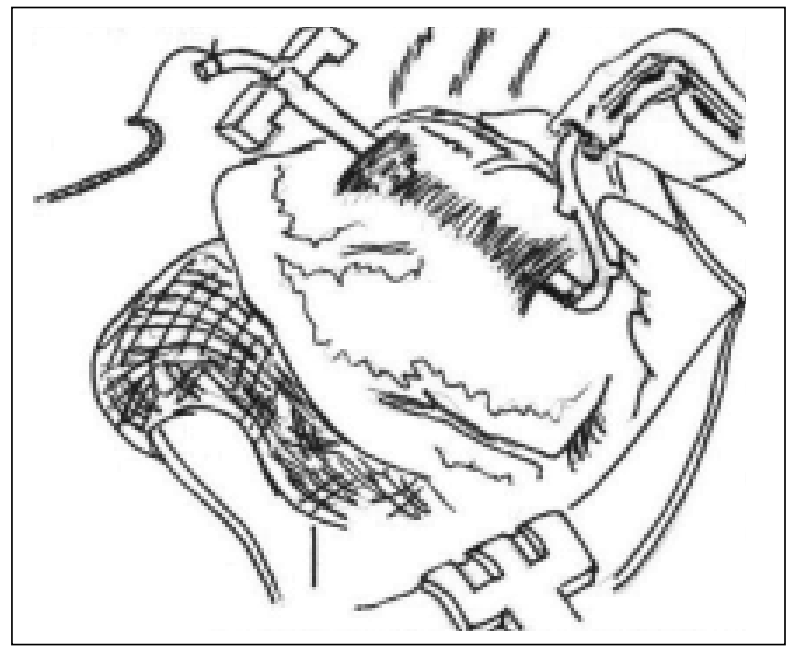

Fig. 5

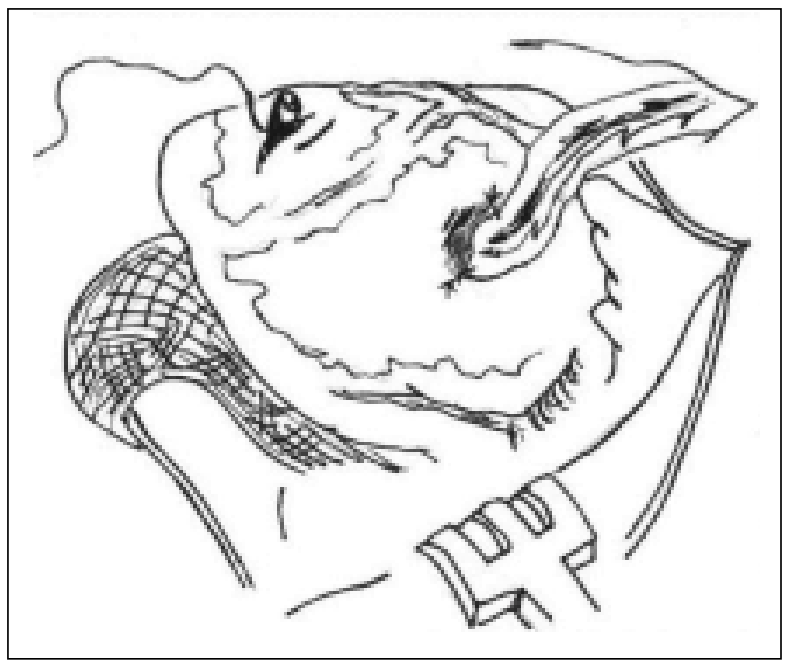

Fig. 6 


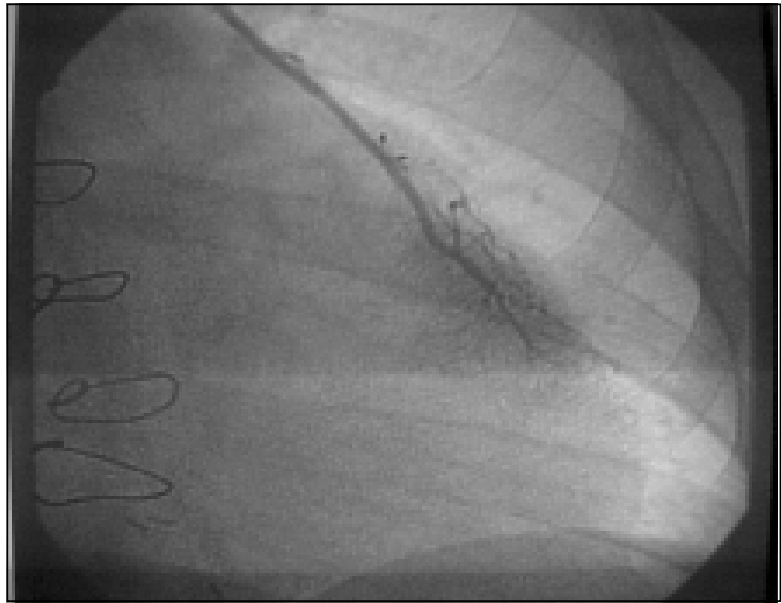

Fig. 7

\section{CONCLUSÃO}

O número de pacientes coronarianos, com comorbidade elevada, de difícil manuseio clínico, principalmente diabéticos, é cada vez maior. São pacientes que geralmente apresentam lesão difusa da artéria DA, com músculo viável em seu território, associada ou não a lesões de outros vasos. Neste subgrupo de pacientes, o procedimento de Vineberg pode ser empregado, associando-se ou não à revascularização direta de outras artérias do sistema coronariano, inclusive as posteriores, sem uso da circulação extracorpórea ${ }^{(8,9)}$.

Nos últimos vinte meses, realizamos vinte e três procedimentos de Vineberg, utilizando a técnica descrita, sem morbimortalidade no pósoperatório imediato. Estudo angiográfico realizado nos 10 primeiros pacientes, após três meses da cirurgia, mostrou perviedade em noventa por cento dos casos, com formação de anastomoses entre a ATIE e os vasos miocárdicos (Figuras 7 e 8 ).

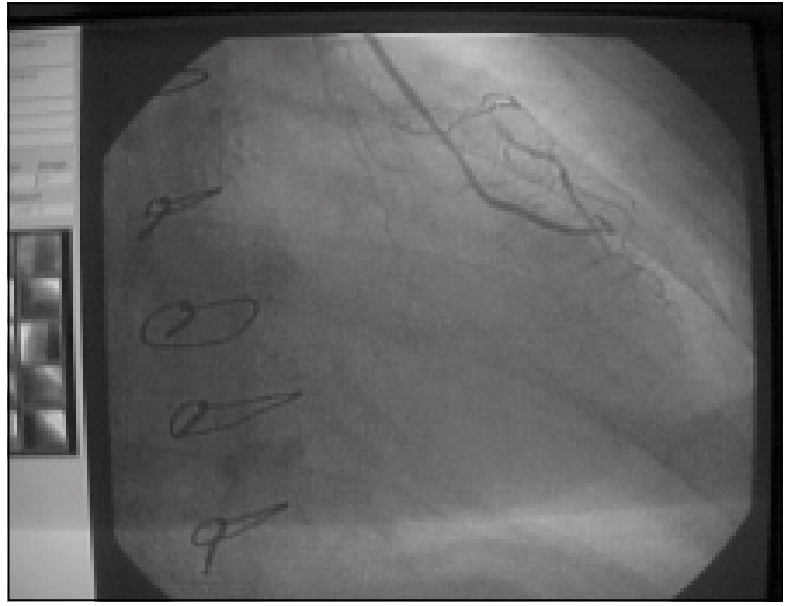

Fig. 8

Podemos, de maneira didática, enumerar as principais vantagens desta nova técnica:

$\Rightarrow$ Rapidez e simplicidade na execução;

$\Rightarrow$ Menor trauma das fibras miocárdicas pelo cateter introdutor, reduzindo a possibilidade de sangramento, infarto e insuficiência cardíaca;

$\Rightarrow$ Aumento de $50 \%$ (de quatro para seis centímetros) do segmento de ATIE implantado (10);

$\Rightarrow$ Alto índice de perviedade do enxerto (90\%);

$\Rightarrow$ Fácil reprodutibilidade.

Por fim, acreditamos que a sistematização desta nova técnica, com a utilização da "bainha introdutora", fará com que o procedimento de Vineberg possa ser realizado com maior freqüência, tanto de maneira isolada, como associado à revascularização direta de outras artérias coronárias, principalmente se a teoria da angiogênese for realmente comprovada no que se refere à doença coronariana.

RBCCV 44205-533

LOBO FILHO, J.G. - Vineberg Procedure: a new technical approach. Rev Bras Cir Cardiovasc 2001; 16(1): 66-9.

ABSTRACT: The objective of this paper is to present a new systematization of the Vineberg Procedure's technique providing a valid and feasible operation. It consists in implanting a long segment of the internal thoracic artery into the ventricular wall utilizing an introductory sheath to create the tunnel.

DESCRIPTORS: Myocardial revascularization, methods. Cardiovascular surgical procedures, methods. Extracorporeal circulation. Thoracic artery, left, surgery. Thoracic artery, left, transplantation. 


\section{REFERÊNCIAS BIBLIOGRÁFICAS}

1 Marx R, Jax TW, Kelm M, Schoebel FC, Strauer BE Vineberg graft: flow reserve of bilateral implantation after 27 years. Ann Thorac Surg 2001; 71: 341-3.

2 Van Langenhove G, Serrano P, Serruys PW - Vineberg revisited long-term survival more than two decades after direct surgical myocardial revascularization. Int J Cardiol 2000; 73: 83-6.

3 Krabatsch T, Grauhan O, Hetzer R - Unilateral Vineberg arterial graft with a patency of 30 years. Circulation 2000; 102: 1724-5.

4 Vineberg A, Afridi S, Sahi S - Direct revascularization of acute myocardial infarction by implantation of left internal mammary artery into infarcted left ventricular myocardium. Surg Ginecol Obst 1975; 140: 44-52.

5 Vineberg A - Evidence that revascularization by ventricularinternal mammary artery implants increases longevity: twenty-four year, nine month follow-up. $J$ Thorac Cardiovasc Surg 1975; 70: 381-97.
6 Sewell W - The surgical treatment of coronary artery disease. Am J Surg 1959; 25: 560.

7 Favaloro RG, Effler DB, Groves LK, Sones FM Jr, Ferguson DJ - Myocardial revascularization by internal mammary artery implant procedures: clinical experience. J Thorac Cardiovasc Surg 1967; 54: 359-70.

8 Lobo Filho JG, Dantas MCBR, Rolim JGV et al. - Cirurgia de revascularização completa do miocárdio sem circulação extracorpórea: uma realidade. Rev Bras Cir Cardiovasc 1997; 12: 115-21.

9 Lobo Filho JG, Albuquerque JMAC, Gomes CBG et al. Revascularização cirúrgica das artérias posteriores do coração sem circulação extracorpórea. Arq Bras Cardiol 1999; 72: 593-600.

10 Vineberg A - Revascularization via healthy myocardial arteriolar networks compared with that through diseased surface coronary arteries. Isr J Med Sci 1975; 11: 250-63. 